\title{
Automatic Conversion of Tables to LongForm Dataframes
}

\author{
by Jimmy $\mathrm{Oh}$
}

\begin{abstract}
TableToLongForm automatically converts hierarchical Tables intended for a human reader into a simple LongForm dataframe that is machine readable, making it easier to access and use the data for analysis. It does this by recognising positional cues present in the hierarchical Table (which would normally be interpreted visually by the human brain) to decompose, then reconstruct the data into a LongForm dataframe. The article motivates the benefit of such a conversion with an example Table, followed by a short user manual, which includes a comparison between the simple one argument call to TableToLongForm, with code for an equivalent manual conversion. The article then explores the types of Tables the package can convert by providing a gallery of all recognised patterns. It finishes with a discussion of available diagnostic methods and future work.
\end{abstract}

\section{Introduction}

TableToLongForm is an R package that automatically converts hierarchical Tables ${ }^{1}$ intended for a human reader into a LongForm $\mathrm{R}$ "data. frame" that is machine readable. ${ }^{2}$ While several tools exist that can aid in manipulation of data, such as OpenRefine (OpenRefine, 2013), which can be used to clean messy data, the speedr R package (Visne et al., 2012), which can aid in filtering data, and $\mathrm{R}$ packages like reshape2 (Wickham, 2007) and plyr (Wickham, 2011), which enable restructuring of the data to focus on specific aspects of the data, for these tools to work their magic you first need machine readable data. However, data released as Tables are not machine readable. At best, such tools will provide some aid in manually converting the data to something that is machine readable, a process that is costly in terms of both time and effort. TableToLongForm is an automatic tool for converting a family of Tables to a machine readable form, and once so converted the user is free to use their favourite tool, $\mathrm{R}$ or otherwise, to make full use of the data.

The article motivates the benefit of such a conversion with an example Table, followed by a short user manual, which includes a comparison between the simple one argument call to TableToLongForm, with code for an equivalent manual conversion. The article then explores the types of Tables the package can convert by providing a gallery of all recognised patterns. It finishes with a discussion of available diagnostic methods and future work.

\section{Motivation}

There is still a prevalence of data releases being made for direct human consumption in formats that are not machine readable, a significant barrier to effective utilisation of the data. One symptom of this is the release of data in tabular form that relies on a hierarchy that can only be understood after identifying patterns and discerning the structure of the Table, a task easy for a human brain but rather difficult for a computer.

An example of such a Table is shown in Figure 1. For such a Table, the computer will be unable to easily read in the data due to the difficulty in finding all information related to a piece of data. Take the number ' 876 ' in cell $(5,9)$ for instance; to collect all the information linked to that number we must look at cell $(5,1)$ for the time period $(' 2007 \mathrm{Q} 4$ '), cell $(4,9)$ for the data heading ('Total Labour Force'), cell $(3,2)$ for the ethnic category ('European Only') and cell $(2,2)$ for the gender category ('Male'). Note that, aside from the time period and the data heading, the other information related to cell $(5,9)$ were neither in the same row nor the same column. The human brain can interpret the positional cues to understand the hierarchy fairly easily; the computer requires a lot more work.

Preparing such data for use would normally require a non-trivial time investment to restructure the data in a manner that can be machine read and used. If such preparatory work was done manually, such work will have to be repeated multiple times as the data is updated. In some cases the data will be spread across multiple files, which means that much more preparatory work. Even if the work is scripted, small changes in the format can easily throw a wrench into the works and break it. All of this quickly adds up to a significant time cost to make use of data released in Tables.

\footnotetext{
${ }^{1}$ Table, with a capital $\mathrm{T}$, is used in this article to specifically mean hierarchical tables, e.g. Figure 1.

${ }^{2}$ Machine readable is used to mean that the format is intended for access and manipulation by computers, and it is thus much easier to use the data for various purposes, such as statistical analysis. It can alternatively be described as Tidy Data (Wickham, 2014), with the conversion taking the data closer to the ideal 'tidy' form.
} 
LongForm is a simple alternative data format that most $\mathrm{R}$ users will find familiar as an $\mathrm{R}$ "data. frame" object, the format that most $\mathrm{R}$ functions require of their data. TableToLongForm automatically converts Tables to LongForm dataframes, ${ }^{3}$ which can mean significant savings in time while enabling much greater utilisation of the data. Figure 2 shows Figure 1 after automatic conversion using TableToLongForm, which took around a tenth of a second on the author's machine. In particular, note that the same data we examined above, ' 876 ' now in cell $(2,11)$, has all related information in the same row (except for the column heading, which is in the same column), making it easy for the computer to understand and manipulate.

\begin{tabular}{|c|c|c|c|c|c|c|c|c|c|c|c|}
\hline & 1 & 2 & 3 & 4 & 5 & 6 & 7 & 8 & 9 & 10 & 11 \\
\hline 1 & Labour F & ce Status by & Sex by Sing & /Comb Ethni & ic Group (Qrt & tly-Mar/Jun/s & Sep/Dec) & & & & \\
\hline 2 & & Male & & & & & & & & & \\
\hline 3 & & European $\mathrm{O}$ & & & & & & & & Maori Only & \\
\hline 4 & & Persons En & Persons Un & Not in Labo & Working $\mathrm{Ag}$ & Labour Forc & Unemploym & Employmen & Total Labou & Persons Em & Persons Un \\
\hline 5 & $2007 Q 4$ & 856 & 20 & 280 & 1,156 & 76 & 2 & 74 & 876 & 71 & 6 \\
\hline 6 & 2008Q1 & 863 & 25 & 284 & 1,172 & 76 & 3 & 74 & 888 & 69 & 8 \\
\hline 7 & 2008Q2 & 850 & 26 & 281 & 1,157 & 76 & 3 & 74 & 876 & 67 & 6 \\
\hline 8 & 2008Q3 & 840 & 30 & 286 & 1,155 & 75 & 3 & 73 & 869 & 72 & 9 \\
\hline 9 & $2008 Q 4$ & 855 & 30 & 275 & 1,159 & 76 & 3 & 74 & 884 & 76 & 8 \\
\hline 10 & 2009Q1 & 845 & 35 & 279 & 1,160 & 76 & 4 & 73 & 880 & 75 & 8 \\
\hline 11 & 2009Q2 & 832 & 35 & 280 & 1,146 & 76 & 4 & 73 & 866 & 74 & 10 \\
\hline 12 & 2009Q3 & 813 & 42 & 290 & 1,146 & 75 & 5 & 71 & 856 & 71 & 11 \\
\hline 13 & 2009Q4 & 831 & 40 & 277 & 1,148 & 76 & 5 & 72 & 871 & 72 & 14 \\
\hline 14 & 2010Q1 & 822 & 36 & 283 & 1,142 & 75 & 4 & 72 & 859 & 72 & 11 \\
\hline
\end{tabular}

Figure 1: An example of a hierarchical Table. The Table is of the Labour Force Status data (Statistics New Zealand, 2013) and in total spans 240 columns. The Table is too large to be immediately useful for humans, and cannot even be easily manipulated with a computer, as understanding the data requires linking information across different rows and columns.

\begin{tabular}{|c|c|c|c|c|c|c|c|c|c|c|c|}
\hline & 1 & 2 & 3 & 4 & 5 & 6 & 7 & 8 & 9 & 10 & 11 \\
\hline 1 & & & & Persons Em & Persons Un & Not in Labo & Working Ag & Labour Forc & Unemploym & Employmer & Total Labou \\
\hline 2 & Male & European C & 2007Q4 & 856 & 20 & 280 & 1,156 & 76 & 2 & 74 & 876 \\
\hline 3 & Male & European $\mathrm{C}$ & 2008Q1 & 863 & 25 & 284 & 1,172 & 76 & 3 & 74 & 888 \\
\hline 4 & Male & European C & $2008 Q 2$ & 850 & 26 & 281 & 1,157 & 76 & 3 & 74 & 876 \\
\hline 5 & Male & European C & $2008 Q 3$ & 840 & 30 & 286 & 1,155 & 75 & 3 & 73 & 869 \\
\hline 6 & Male & European $\mathrm{C}$ & $2008 Q 4$ & 855 & 30 & 275 & 1,159 & 76 & 3 & 74 & 884 \\
\hline 7 & Male & European $\mathrm{C}$ & 2009Q1 & 845 & 35 & 279 & 1,160 & 76 & 4 & 73 & 880 \\
\hline 8 & Male & European C & $2009 Q 2$ & 832 & 35 & 280 & 1,146 & 76 & 4 & 73 & 866 \\
\hline 9 & Male & European $\mathrm{C}$ & 2009Q3 & 813 & 42 & 290 & 1,146 & 75 & 5 & 71 & 856 \\
\hline 10 & Male & European $\mathrm{C}$ & 2009Q4 & 831 & 40 & 277 & 1,148 & 76 & 5 & 72 & 871 \\
\hline 11 & Male & European C & 2010Q1 & 822 & 36 & 283 & 1,142 & 75 & 4 & 72 & 859 \\
\hline 12 & Male & European $\mathrm{C}$ & 2010Q2 & 825 & 40 & 290 & 1,155 & 75 & 5 & 71 & 865 \\
\hline 13 & Male & European $\mathrm{C}$ & 2010Q3 & 837 & 31 & 287 & 1,155 & 75 & 4 & 72 & 868 \\
\hline 14 & Male & European C & 2010Q4 & 838 & 40 & 277 & 1,155 & 76 & 4 & 73 & 878 \\
\hline
\end{tabular}

Figure 2: An example of a LongForm dataframe. This is the Table in Figure 1 after automatic conversion with TableToLongForm and in total spans 660 rows. Now all related information can be found in the same row or column, making the data much more useful.

\section{User manual}

\section{Loading the data}

TableToLongForm's preferred argument is a "matrix" of mode "character". If a "data.frame" is supplied instead, it is coerced to a "matrix" with a warning. Empty cells should be classed as "NA" for correct operation of the algorithms. Currently TableToLongForm does not distinguish between missing values and empty space, both are treated as "NA" values.

As the Labour Force Status data used in Figure 1 classifies missing values as '. .', we must ensure $\mathrm{R}$ correctly reads these, in addition to empty cells, as "NA" values. ${ }^{4}$

$$
\begin{array}{r}
\text { LabourForce = as.matrix }(\text { read. } \operatorname{csv}(\text { "StatsNZLabourForce.csv", } \\
\text { header }=\text { FALSE, na.strings }=c(" \text { " , ". "))) }
\end{array}
$$

\footnotetext{
${ }^{3}$ I use the term LongForm loosely and in some cases TableToLongForm will result in WideForm output as the difference can depend on contextual information the computer cannot comprehend. However, the output will be machine readable and many tools, such as those mentioned in the opening paragraph, can be used to further reformat the data, including conversions between LongForm and WideForm.

"This Table, after being read in as a "matrix" as shown, is included in TableToLongForm as part of data(TCData), and can be accessed with TCData[["StatsNZLabourForce"]]
} 


\section{Calling TableToLongForm}

If the Table can be recognised by TableToLongForm, a simple call to TableToLongForm with just a single argument is all that is needed. TableToLongForm has additional optional arguments used primarily for diagnostic purposes, which are covered in the diagnostics section at the end of the article.

LabourForce . converted $=$ TableToLongForm(LabourForce)

\section{Aside: manual conversion}

For comparison the code for manual conversion of the table is provided below. We note after careful observation of the data that:

- There are 3 gender categories: 'Male', 'Female' and 'Total Both Sexes', each 80 columns in width.

- There are 10 ethnic categories, each a consistent 8 columns in width.

- The data are found in rows 5 to 26 .

Armed with this knowledge, we can write the above code that, with some trial and error and cross-checking of results, will successfully convert the Table to a LongForm. This code is fairly compact and efficiency-wise beats TableToLongForm, taking a little over a thousandth of a second to make the conversion (compared to about a hundredth of a second for a call to TableToLongForm) on the author's machine. However, it took a non-trivial investment of time to code and test the results (it took the author about 30 minutes), is mostly useless for any other Table, and if any of the many strong assumptions it makes are violated (e.g. a new row of data is added), it breaks and requires fixing, which means even more time consumed. All this work and hassle to just read in the data in a useful format.

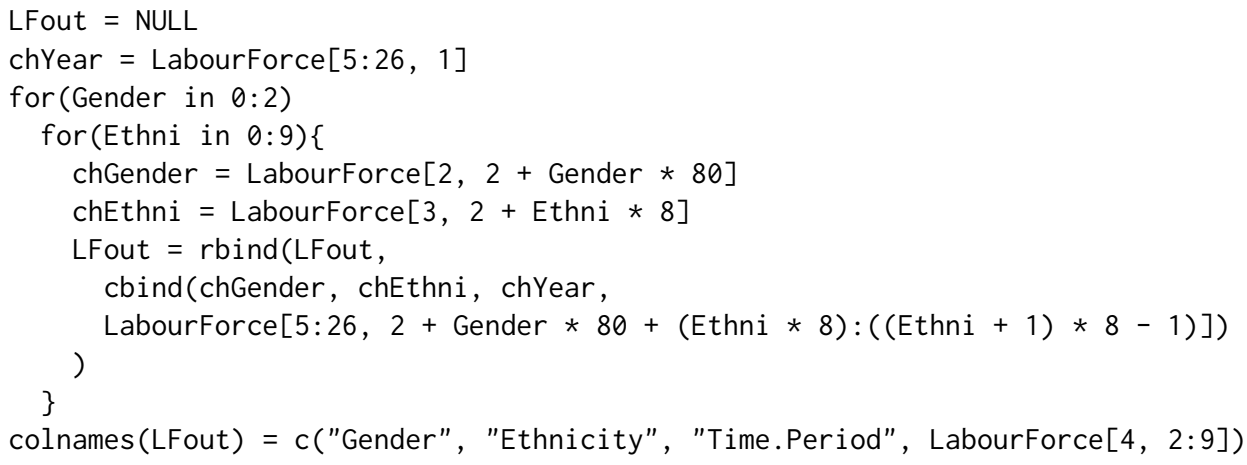

\section{IdentResult}

For a successful conversion, TableToLongForm must first find the Table, that is, it must Identify the rows and columns in which the labels and data values can be found. This task can be surprisingly difficult, requiring many fringe-case checks and exception handling. The current core identification algorithm searches for blocks (rectangular regions) of numbers in the supplied "matrix". This region is assumed to contain the data and from it TableToLongForm infers the locations of the corresponding labels. The result, after some extra work to handle fringe-cases and the like, is the IdentResult, a "list" which specifies the rows and columns in which the labels and the data can be found.

If TableToLongForm fails to correctly Identify the correct rows and columns, it is possible to manually specify the IdentResult as an argument. This is the case for the Table in Figure 3, where one of the row label columns is a Year column consisting only of numbers. TableToLongForm's numeric label detection algorithm is still quite primitive and fails to correctly identify column 3 as a label, but by manually specifying the IdentResult, TableToLongForm can still successfully convert the Table; the resulting "data. frame" is shown in Figure 4. Even for cases such as this where the IdentResult must be manually specified, the work required for the conversion with TableToLongForm will be strictly less than for a manual conversion as we would need the same information, and more, to convert manually.

TableToLongForm(NEET, IdentResult $=\operatorname{list}($ rows $=\operatorname{list}($ label $=3: 4$, data $=5: 46)$,

cols $=$ list $($ label $=2: 3$, data $=4: 24))$ ) 


\begin{tabular}{|c|c|c|c|c|c|c|c|c|c|c|c|}
\hline & & 2 & 3 & 4 & 5 & 6 & $7 \mid$ & 8 & 9 & 10 & 11 \\
\hline 1 & \multicolumn{11}{|c|}{ TABLE 1: (a) Number of 16-24 year olds Not in Education, Employment or Training (NEET) and (b) associated Confidence Intervals b! } \\
\hline \multicolumn{12}{|l|}{2} \\
\hline 3 & & & & \multicolumn{4}{|l|}{ (a) Number } & & & & \\
\hline 4 & & \multicolumn{2}{|c|}{ Quarterly LFS series } & England & North East & North West & Yorks \& Hur & East Midlan & West Midlaı & East of Eng & London \\
\hline 5 & & Q2 & 2000 & 652,000 & 61,000 & 92,000 & 72,000 & 60,000 & 75,000 & 53,000 & 127,000 \\
\hline 6 & & Q3 & 2000 & 750,000 & 57,000 & 113,000 & 87,000 & 69,000 & 89,000 & 64,000 & 131,000 \\
\hline 7 & & Q4 & 2000 & 629,000 & 48,000 & 97,000 & 72,000 & 57,000 & 84,000 & 55,000 & 93,000 \\
\hline 8 & & Q1 & 2001 & 667,000 & 53,000 & 114,000 & 77,000 & 58,000 & 82,000 & 61,000 & 100,000 \\
\hline 9 & & Q2 & 2001 & 650,000 & 42,000 & 112,000 & 77,000 & 53,000 & 75,000 & 62,000 & 111,000 \\
\hline 10 & & Q3 & 2001 & 774,000 & 50,000 & 132,000 & 84,000 & 60,000 & 79,000 & 75,000 & 140,000 \\
\hline 11 & & Q4 & 2001 & 660,000 & 46,000 & 110,000 & 75,000 & 50,000 & 79,000 & 60,000 & 116,000 \\
\hline 12 & & Q1 & 2002 & 699,000 & 51,000 & 114,000 & 83,000 & 59,000 & 88,000 & 61,000 & 111,000 \\
\hline 13 & & Q2 & 2002 & 703,000 & 45,000 & 117,000 & 84,000 & 55,000 & 85,000 & 61,000 & 123,000 \\
\hline 14 & & Q3 & 2002 & 795,000 & 49,000 & 115,000 & 111,000 & 58,000 & 96,000 & 71,000 & 143,000 \\
\hline
\end{tabular}

Figure 3: Another example of a hierarchical Table. The Table is of the NEET statistics (Department for Education (UK), 2013) and is relatively tame in terms of complexity. The work required to manually convert and read in such a Table would be light, but still enough to be an annoying time sink. Highlighted are the three regions TableToLongForm must identify for successful conversion, and if automatic identification of these regions fail, the rows and columns corresponding to these three rectangular regions can be specified manually.

\begin{tabular}{|c|c|c|c|c|c|c|c|c|c|c|c|}
\hline & 1 & 2 & 3 & 4 & 5 & 6 & 7 & 8 & 9 & 10 & 11 \\
\hline 1 & & & & England & North East & North West & Yorks \& Hu & East Midlan & West Midla & East of Eng & London \\
\hline 2 & (a) Number & Q2 & 2000 & 652,000 & 61,000 & 92,000 & 72,000 & 60,000 & 75,000 & 53,000 & 127,000 \\
\hline 3 & (a) Number & Q3 & 2000 & 750,000 & 57,000 & 113,000 & 87,000 & 69,000 & 89,000 & 64,000 & 131,000 \\
\hline 4 & (a) Number & Q4 & 2000 & 629,000 & 48,000 & 97,000 & 72,000 & 57,000 & 84,000 & 55,000 & 93,000 \\
\hline 5 & (a) Number & Q1 & 2001 & 667,000 & 53,000 & 114,000 & 77,000 & 58,000 & 82,000 & 61,000 & 100,000 \\
\hline 6 & (a) Number & Q2 & 2001 & 650,000 & 42,000 & 112,000 & 77,000 & 53,000 & 75,000 & 62,000 & 111,000 \\
\hline 7 & (a) Number & Q3 & 2001 & 774,000 & 50,000 & 132,000 & 84,000 & 60,000 & 79,000 & 75,000 & 140,000 \\
\hline 8 & (a) Number & Q4 & 2001 & 660,000 & 46,000 & 110,000 & 75,000 & 50,000 & 79,000 & 60,000 & 116,000 \\
\hline 9 & (a) Number & Q1 & 2002 & 699,000 & 51,000 & 114,000 & 83,000 & 59,000 & 88,000 & 61,000 & 111,000 \\
\hline 10 & (a) Number & Q2 & 2002 & 703,000 & 45,000 & 117,000 & 84,000 & 55,000 & 85,000 & 61,000 & 123,000 \\
\hline 11 & (a) Number & Q3 & 2002 & 795,000 & 49,000 & 115,000 & 111,000 & 58,000 & 96,000 & 71,000 & 143,000 \\
\hline 12 & (a) Number & Q4 & 2002 & 660,000 & 49,000 & 100,000 & 74,000 & 55,000 & 77,000 & 69,000 & 113,000 \\
\hline 13 & (a) Number & Q1 & 2003 & 730,000 & 51,000 & 99,000 & 95,000 & 54,000 & 90,000 & 78,000 & 122,000 \\
\hline 14 & (a) Number & Q2 & 2003 & 709,000 & 51,000 & 107,000 & 87,000 & 59,000 & 88,000 & 66,000 & 112,000 \\
\hline
\end{tabular}

Figure 4: Another example of a LongForm dataframe. This is the Table in Figure 3 after automatic conversion with TableToLongForm. Although the conversion required the aid of a human to specify the optional argument IdentResult to be successful, the work required with TableToLongForm will be strictly less than for a manual conversion as we would need the same information, and more, to convert manually.

\section{Recognised patterns}

TableToLongForm consists of a number of algorithms that can collectively process a variety of socalled recognised patterns of hierarchical structure (also called the parentage of the labels). Any Table that consists of some combination of the recognised patterns can be automatically converted with TableToLongForm. It is not strictly necessary for a user to know what the patterns are, as they can simply try calling TableToLongForm on the Table to see if it converts. All the recognised patterns are listed here for reference. ${ }^{5}$ For an example of a real Table that demonstrates a combination of the recognised patterns, refer to Real Example - NZQA located at the end of this section.

For each pattern an example table is first shown using toy data, that displays the pattern, followed by a short description of the pattern, and ending with the example table converted with TableToLongForm.

Many of the recognised patterns apply only for row labels. Column labels are recognised by noticing that the transpose of column labels can often be processed as row labels, though there are several fringe cases that must be corrected for.

\footnotetext{
${ }^{5}$ All the Tables demonstrating the recognised patterns are included in TableToLongForm as part of data(TCData). TableToLongForm can be called on these Tables for the converted versions, e.g. TableToLongForm(TCData[["ToyExByEmptyBelow"]])
} 


\section{Empty Below}

\begin{tabular}{|l|l|l|r|r|r|r}
\hline & 1 & \multicolumn{1}{|c|}{2} & \multicolumn{1}{c|}{3} & \multicolumn{2}{c|}{4} & \multicolumn{2}{c}{5} \\
\hline 1 & & & Column 1 & Column 2 & Column 3 & \multicolumn{1}{c}{ Column 4 } \\
\hline 2 & Row Parent1 & Row Child1 & 10 & 20 & 30 & 40 \\
\hline 3 & & Row Child2 & 11 & 21 & 31 & 41 \\
\hline 4 & Row Parent2 & Row Child1 & 12 & 22 & 32 & 42 \\
\hline 5 & & Row Child2 & 13 & 23 & 33 & 43 \\
\hline
\end{tabular}

Above, we have an example of the Empty Below pattern, the most simple type of parentage. Here the parent and children are in different columns and we can see which of the children belong to which parent through the use of empty space below each parent. The Table after conversion to a LongForm follows.

\begin{tabular}{|c|c|c|c|c|c|c|}
\hline & 1 & 2 & 3 & 4 & 5 & 6 \\
\hline 1 & & & Column 1 & Column 2 & Column 3 & Column 4 \\
\hline 2 & Row Parent1 & Row Child1 & 10 & 20 & 30 & 40 \\
\hline 3 & Row Parent1 & Row Child2 & 11 & 21 & 31 & 41 \\
\hline 4 & Row Parent2 & Row Child1 & 12 & 22 & 32 & 42 \\
\hline 5 & Row Parent2 & Row Child2 & 13 & 23 & 33 & 43 \\
\hline
\end{tabular}

\section{Empty Right 1}

\begin{tabular}{|c|c|c|c|c|c|c|}
\hline & 1 & 2 & 4 & 5 & 6 & 7 \\
\hline 1 & & & Column 1 & Column 2 & Column 3 & Column 4 \\
\hline 2 & Row Parent1 & & 10 & 20 & 30 & 40 \\
\hline 3 & Row Child1 & Row Child-Child1 & 11 & 21 & 31 & 41 \\
\hline 4 & Row Child2 & Row Child-Child2 & 12 & 22 & 32 & 42 \\
\hline 5 & Row Parent2 & & 13 & 23 & 33 & 43 \\
\hline 6 & Row Child1 & Row Child-Child1 & 14 & 24 & 34 & 44 \\
\hline 7 & & Row Child-Child2 & 15 & 25 & 35 & 45 \\
\hline
\end{tabular}

Above, we have an example of the most basic form of the Empty Right pattern. In this situation we have children in the same column as their parent. We can still recognise these as children if the children have children (Child-Child) in a different column, while the parent does not (and hence the parent is Empty Right). Note the values pertaining to the Parent (if any) are discarded. This is because they are assumed to simply represent the sum of their children's values. The Table after conversion to a LongForm follows.

\begin{tabular}{|c|c|c|c|c|c|c|c|}
\hline & 1 & 2 & 3 & 4 & 5 & 6 & 7 \\
\hline 1 & & & & Column 1 & Column 2 & Column 3 & Column 4 \\
\hline 2 & Row Parent1 & Row Child1 & Row Child-Cr & 11 & 21 & 31 & 41 \\
\hline 3 & Row Parent1 & Row Child2 & Row Child-Cr & 12 & 22 & 32 & 42 \\
\hline 4 & Row Parent2 & Row Child1 & Row Child-Cr & 14 & 24 & 34 & 44 \\
\hline 5 & Row Parent2 & Row Child1 & Row Child-Ch & 15 & 25 & 35 & 45 \\
\hline
\end{tabular}

\section{Empty Right 2}

\begin{tabular}{|c|c|c|c|c|c|c|}
\hline & 1 & 2 & 3 & 4 & 5 & 6 \\
\hline 1 & & & Column 1 & Column 2 & Column 3 & Column 4 \\
\hline 2 & Row Parent1 & & 10 & 20 & 30 & 40 \\
\hline 3 & & Row Child1 & 11 & 21 & 31 & 41 \\
\hline 4 & & Row Child2 & 12 & 22 & 32 & 42 \\
\hline 5 & Row Parent2 & & 13 & 23 & 33 & 43 \\
\hline 6 & & Row Child1 & 14 & 24 & 34 & 44 \\
\hline 7 & & Row Child2 & 15 & 25 & 35 & 45 \\
\hline
\end{tabular}

Above, we have an example of both Empty Below and Empty Right. Either algorithm can handle this situation, but simply due to the ordering of the algorithms such situations are handled as Empty Right. The Table after conversion to a LongForm follows. 


\begin{tabular}{|c|c|c|c|c|c|c|}
\hline & 1 & 2 & 3 & 4 & 5 & 6 \\
\hline 1 & & & Column 1 & Column 2 & Column 3 & Column 4 \\
\hline 2 & Row Parent1 & Row Child1 & 11 & 21 & 31 & 41 \\
\hline 3 & Row Parent1 & Row Child2 & 12 & 22 & 32 & 42 \\
\hline 4 & Row Parent2 & Row Child1 & 14 & 24 & 34 & 44 \\
\hline 5 & Row Parent2 & Row Child2 & 15 & 25 & 35 & 45 \\
\hline
\end{tabular}

\section{Empty Right 3}

\begin{tabular}{|c|c|c|c|c|c|c|c|c|}
\hline & 1 & 2 & 3 & 4 & 5 & 6 & 7 & 8 \\
\hline 1 & & & & Column 1 & Column 2 & Column 3 & Column 4 & \\
\hline 2 & \multicolumn{2}{|c|}{ Row Super-Parent1 } & & 10 & 20 & 30 & 40 & \\
\hline 3 & Row Parent1 & & & 11 & 21 & 31 & 41 & \\
\hline 4 & Row Child1 & \multicolumn{2}{|c|}{ Row Child-Child1 } & 12 & 22 & 32 & 42 & \\
\hline 5 & Row Parent2 & & & 13 & 23 & 33 & 43 & \\
\hline 6 & Row Child1 & \multicolumn{2}{|c|}{ Row Child-Child1 } & 14 & 24 & 34 & 44 & \\
\hline 7 & \multicolumn{2}{|c|}{ Row Super-Parent2 } & & 15 & 25 & 35 & 45 & \\
\hline 8 & Row Parent1 & & & 16 & 26 & 36 & 46 & \\
\hline 9 & Row Child1 & \multicolumn{2}{|c|}{ Row Child-Child1 } & 17 & 27 & 37 & 47 & \\
\hline 10 & Row Parent2 & & & 18 & 28 & 38 & 48 & \\
\hline 11 & Row Child1 & \multicolumn{2}{|c|}{ Row Child-Child1 } & 19 & 29 & 39 & 49 & \\
\hline
\end{tabular}

Above, we have an example of a complex version of the Empty Right pattern. The "parent-child in the same column" situation has been extended further and we now have parents (Super-Parent) who have children (Parent), who each further have children (Child), all in the same column. Such situations can still be recognised if the lowest-level children in the column (Child) have children in a different column (Child-Child), while its direct parents (Parent) each have children in the same column (Child) but not in a different column (is Empty Right), and the top-most parents (Super-Parents) also have no children in a different column (is also Empty Right). The algorithm cannot currently handle super-super-parents. The Table after conversion to a LongForm follows.

\begin{tabular}{|c|c|c|c|c|c|c|c|c|}
\hline & 1 & 2 & 3 & 4 & 5 & 6 & 7 & 8 \\
\hline 1 & & & & & Column 1 & Column 2 & Column 3 & Column 4 \\
\hline 2 & Row Super-P & Row Parent1 & Row Child1 & Row Child-Cr & 12 & 22 & 32 & 42 \\
\hline 3 & Row Super-P & Row Parent2 & Row Child1 & Row Child-Cr & 14 & 24 & 34 & 44 \\
\hline 4 & Row Super-P & Row Parent1 & Row Child1 & Row Child-Cr & 17 & 27 & 37 & 47 \\
\hline 5 & Row Super-P & Row Parent2 & Row Child1 & Row Child-Cr & 19 & 29 & 39 & 49 \\
\hline
\end{tabular}

\section{Multi-row Column Label}

\begin{tabular}{|c|c|c|c|c|c|c|}
\hline & 1 & 2 & 3 & 4 & 5 & 6 \\
\hline 1 & & Column & Column & Column & Column & \\
\hline 2 & & Child1 & Child2 & Child3 & Child4 & \\
\hline 3 & Row 1 & 10 & 20 & 30 & 40 & \\
\hline 4 & Row 2 & 11 & 21 & 31 & 41 & \\
\hline 5 & Row 3 & 12 & 22 & 32 & 42 & \\
\hline 6 & Row 4 & 13 & 23 & 33 & 43 & \\
\hline
\end{tabular}

Above, we have an example of Multi-row Column Labels. Often column labels are physically split over multiple rows rather than making use of line breaks in the same cell. In such occurrences, any row not identified as a parent are collapsed into a single row of labels. The Table after conversion to a LongForm follows.

\begin{tabular}{|l|l|r|r|r|r|r}
\hline & 1 & 2 & 3 & \multicolumn{2}{c|}{5} & \multicolumn{2}{c|}{5} \\
\hline 1 & & Column Child & Column Child; & Column Child & Column Child4 \\
\hline 2 & Row 1 & 10 & 20 & 30 & 40 & \\
\hline 3 & Row 2 & 11 & 21 & 31 & 41 & \\
\hline 4 & Row 3 & 12 & 22 & 32 & 42 & \\
\hline 5 & Row 4 & 13 & 23 & 33 & 43 & \\
\hline
\end{tabular}




\section{Mismatched Column Label}

\begin{tabular}{|c|c|c|c|c|c|c|c|c|c|}
\hline & 1 & 2 & 3 & 4 & 5 & 6 & 7 & 8 & 9 \\
\hline 1 & & Col Child1 & & Col Child2 & & Col Child3 & & Col Child4 & \\
\hline 2 & Row 1 & & 10 & & 20 & & 30 & & 40 \\
\hline 3 & Row 2 & & 11 & & 21 & & 31 & & 41 \\
\hline 4 & Row 3 & & 12 & & 22 & & 32 & & 42 \\
\hline 5 & Row 4 & & 13 & & 23 & & 33 & & 43 \\
\hline
\end{tabular}

Above, we have an example of Mismatched Column Labels. Sometimes the column labels are in a different column to the data, usually due to a misguided attempt at visual alignment of labels to the data. As long as the correct rows and columns were identified for the data and the labels (see User manual subsection on IdentResult), and if there are the same number of data columns as label columns, these mismatched column labels will be paired with the data columns. The Table after conversion to a LongForm follows.

\begin{tabular}{|c|c|c|c|c|c|c|c|c|c|}
\hline & 1 & 2 & 3 & 4 & 5 & 6 & 7 & 8 & 9 \\
\hline 1 & & Col Child1 & Col Child2 & Col Child3 & Col Child4 & & & & \\
\hline 2 & Row 1 & 10 & 20 & 30 & 40 & & & & \\
\hline 3 & Row 2 & 11 & 21 & 31 & 41 & & & & \\
\hline 4 & Row 3 & 12 & 22 & 32 & 42 & & & & \\
\hline 5 & Row 4 & 13 & 23 & 33 & 43 & & & & \\
\hline
\end{tabular}

\section{Misaligned Column Label}

\begin{tabular}{|c|c|c|c|c|c|c|c|c|c|}
\hline & 1 & 2 & 3 & 4 & 5 & 6 & 7 & 8 & 9 \\
\hline 1 & & & Col Parent1 & & & & Col Parent2 & & \\
\hline 2 & & Col Child1 & Col Child2 & Col Child3 & Col Child4 & Col Child1 & Col Child2 & Col Child3 & Col Child4 \\
\hline 3 & Row 1 & 10 & 20 & 30 & 40 & 50 & 60 & 70 & 80 \\
\hline 4 & Row 2 & 11 & 21 & 31 & 41 & 51 & 61 & 71 & 81 \\
\hline 5 & Row 3 & 12 & 22 & 32 & 42 & 52 & 62 & 72 & 82 \\
\hline 6 & Row 4 & 13 & 23 & 33 & 43 & 53 & 63 & 73 & 83 \\
\hline
\end{tabular}

Above, we have an example of Misaligned Column Labels. Often column parents are physically centred over their children (N.B. where a spreadsheet's cell-merge feature is used to do the centering, the actual value is usually stored in the top-left cell and hence causes no problems). TableToLongForm makes use of pattern recognition to identify repeating patterns in the labels, or in empty cells surrounding the labels, to correct for the misalignment. For the Column Parents row, we find (starting from column 2, the first data column) a pattern of Empty-NonEmpty-Empty-Empty, with the pattern occurring twice. In the Col Child row, we also find a pattern of length 4 occurring twice. This can be used to correctly align the Column Parents to its children. The Table after conversion to a LongForm follows.

\begin{tabular}{|c|c|c|c|c|c|c|c|c|c|}
\hline & 1 & 2 & 3 & 4 & 5 & 6 & 7 & 8 & 9 \\
\hline 1 & & & Col Child1 & Col Child2 & Col Child3 & Col Child4 & & & \\
\hline 2 & Col Parent1 & Row 1 & 10 & 20 & 30 & 40 & & & \\
\hline 3 & Col Parent1 & Row 2 & 11 & 21 & 31 & 41 & & & \\
\hline 4 & Col Parent1 & Row 3 & 12 & 22 & 32 & 42 & & & \\
\hline 5 & Col Parent1 & Row 4 & 13 & 23 & 33 & 43 & & & \\
\hline 6 & Col Parent2 & Row 1 & 50 & 60 & 70 & 80 & & & \\
\hline 7 & Col Parent2 & Row 2 & 51 & 61 & 71 & 81 & & & \\
\hline 8 & Col Parent2 & Row 3 & 52 & 62 & 72 & 82 & & & \\
\hline 9 & Col Parent2 & Row 4 & 53 & 63 & 73 & 83 & & & \\
\hline
\end{tabular}

\section{Misaligned Column Label 2}

\begin{tabular}{|c|c|c|c|c|c|c|c|c|c|}
\hline & 1 & 2 & 3 & 4 & 5 & 6 & 7 & 8 & 9 \\
\hline 1 & & & & Col Super-Pa & rent & & & & \\
\hline 2 & & & Col Parent1 & & & & Col Parent2 & & \\
\hline 3 & & Col Child1 & Col Child2 & Col Child3 & Col Child4 & Col Child1 & Col Child2 & Col Child3 & Col Child4 \\
\hline 4 & Row 1 & 10 & 20 & 30 & 40 & 50 & 60 & 70 & 80 \\
\hline 5 & Row 2 & 11 & 21 & 31 & 41 & 51 & 61 & 71 & 81 \\
\hline 6 & Row 3 & 12 & 22 & 32 & 42 & 52 & 62 & 72 & 82 \\
\hline 7 & Row 4 & 13 & 23 & 33 & 43 & 53 & 63 & 73 & 83 \\
\hline
\end{tabular}

Above, we have a generalised example of Misaligned Column Labels. We now have Column SuperParent which is misaligned to both its direct children, the Column Parents, and to the lowest-level children. The Table after conversion to a LongForm follows. 


\begin{tabular}{|c|c|c|c|c|c|c|c|c|c|}
\hline & 1 & 2 & 3 & 4 & 5 & 6 & 7 & 8 & 9 \\
\hline 1 & & & & Col Child1 & Col Child2 & Col Child3 & Col Child4 & & \\
\hline 2 & Col Super-Pa & Col Parent1 & Row 1 & 10 & 20 & 30 & 40 & & \\
\hline 3 & Col Super-Pa & Col Parent1 & Row 2 & 11 & 21 & 31 & 41 & & \\
\hline 4 & Col Super-Pa & Col Parent1 & Row 3 & 12 & 22 & 32 & 42 & & \\
\hline 5 & Col Super-Pa & Col Parent1 & Row 4 & 13 & 23 & 33 & 43 & & \\
\hline 6 & Col Super-Pa & Col Parent2 & Row 1 & 50 & 60 & 70 & 80 & & \\
\hline 7 & Col Super-Pa & Col Parent2 & Row 2 & 51 & 61 & 71 & 81 & & \\
\hline 8 & Col Super-Pa & Col Parent2 & Row 3 & 52 & 62 & 72 & 82 & & \\
\hline 9 & Col Super-Pa & Col Parent2 & Row 4 & 53 & 63 & 73 & 83 & & \\
\hline
\end{tabular}

\section{Real Example - NZQA}

\begin{tabular}{|c|c|c|c|c|c|c|c|c|c|c|c|c|c|}
\hline & 1 & 2 & 3 & 4 & 5 & 6 & 7 & 8 & 9 & 10 & 11 & 12 & 13 \\
\hline 1 & Scholarship & Entries and & Results by $c$ & Gender and & Ethnicity (Br & roken do & by Decile) & & & & & & \\
\hline 2 & & & & & & & & & & & & & \\
\hline 3 & & & & Decile 1-3 & & & & & & & & & Decile 4-7 \\
\hline 4 & & & \# of & $\#$ & \# & & \# & \# Not & \# & \# & & \# of & \# \\
\hline 5 & Results & & Entries & Absent & SNA & & Assessed & Achieved & Scholarship & Outstanding & & Entries & Absent \\
\hline 6 & & & & & & & & & & & & & \\
\hline 7 & All Subjects & & 714 & 148 & 13 & & 553 & 462 & 81 & 10 & & 6,482 & 1,772 \\
\hline 8 & & & & & & & & & & & & & \\
\hline 9 & Accounting & & 22 & 4 & 0 & ) & 18 & 16 & 2 & 0 & & 156 & 41 \\
\hline 10 & NZ Maori & Male & 2 & 1 & 0 & ) & 1 & 1 & 0 & 0 & & 2 & 1 \\
\hline 11 & & Female & 0 & 0 & 0 & & 0 & 0 & 0 & 0 & & 7 & 2 \\
\hline 12 & NZ Europe: & Male & 2 & 0 & 0 & 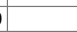 & 2 & 1 & 1 & 0 & & 51 & 13 \\
\hline 13 & & Female & 3 & 0 & 0 & ) & 3 & 2 & 1 & 0 & & 44 & 12 \\
\hline 14 & & Unknown & 0 & 0 & 0 & ) & 0 & 0 & 0 & 0 & & 0 & 0 \\
\hline 15 & Pasifika Per & Male & 2 & 0 & 0 & & 2 & 2 & 0 & 0 & & 3 & 0 \\
\hline 16 & & Female & 6 & 2 & 0 & & 4 & 4 & 0 & 0 & & 4 & 2 \\
\hline 17 & Asian & Male & 5 & 0 & 0 & ) & 5 & 5 & 0 & 0 & & 29 & 4 \\
\hline 18 & & Female & 2 & 1 & 0 & & 1 & 1 & 0 & 0 & & 15 & 7 \\
\hline 19 & Other/Unsp & Male & 0 & 0 & 0 & & 0 & 0 & 0 & 0 & & 0 & 0 \\
\hline 20 & & Female & 0 & 0 & 0 & ) & 0 & 0 & 0 & 0 & & 1 & 0 \\
\hline 21 & & & & & & & & & & & & & \\
\hline 22 & Agricultural & \& Horticultu & 0 & 0 & 0 & ) & 0 & 0 & 0 & 0 & & 15 & 3 \\
\hline 23 & NZ Maori & Male & 0 & 0 & 0 & ) & 0 & 0 & 0 & 0 & & 0 & 0 \\
\hline 24 & & Female & 0 & 0 & 0 & ) & 0 & 0 & 0 & 0 & & 0 & 0 \\
\hline 25 & NZ Europe: & Male & 0 & 0 & 0 & ) & 0 & 0 & 0 & 0 & & 10 & 1 \\
\hline
\end{tabular}

Above, we have an example of real data released in a Table ${ }^{6}$ that demonstrates a combination of many of the patterns listed above. The data comes from the New Zealand Qualifications Authority (NZQA, 2012) regarding the entries and results for their Scholarship Exams. The Table demonstrates Empty Below, Empty Right (including Type 3, as 'All Subjects' is a super-parent), Multi-row Column Labels, and Misaligned Column Labels. The Table is substantially more complex than the Labour Force Status data used in Figure 1, and will require considerably more work to convert manually. Worse, each year of the data is stored in a separate Table, each with slight differences in format. Thus the manual conversion code would have to either be individually tweaked for each year (requiring yet more work), or be flexible enough to handle these differences (requiring substantially more work). Other data from NZQA faces bigger problems; though the Scholarships data can all be obtained in a mere 8 Tables (for 8 years from 2004 to 2011), the Subjects data not only requires a separate Table for each year, but also for each subject (of which there are 91). Obtaining the greatest breakdown possible for the Subjects data across all other variables requires thousands of individual Tables. Without automatic conversion with TableToLongForm, simply reading in such data for use would require too much work to be practical. The Table after conversion to a LongForm follows.

\begin{tabular}{|c|c|c|c|c|c|c|c|c|c|c|c|c|c|}
\hline & 1 & 2 & 3 & 4 & 5 & 6 & 7 & 8 & 9 & 10 & 11 & 12 & 13 \\
\hline 1 & & & & & & \# of Entries \# & Absent & \# SNA & \# Assessed & \# Not Achie\# & Scholars & \# Outstandin & \\
\hline 2 & Decile 1-3 & All Subjects & Accounting & NZ Maori & Male & 2 & 1 & 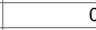 & 1 & 1 & 0 & 0 & \\
\hline 3 & Decile 1-3 & All Subjects & Accounting & NZ Maori & Female & 0 & 0 & 0 & 0 & 0 & 0 & 0 & \\
\hline 4 & Decile 1-3 & All Subjects & Accounting & NZ Europea & Male & 2 & 0 & 0 & 2 & 1 & 1 & 0 & \\
\hline 5 & Decile 1-3 & All Subjects & Accounting & NZ Europea & Female & 3 & 0 & 0 & 3 & 2 & 1 & 0 & \\
\hline 6 & Decile 1-3 & All Subjects & Accounting & NZ Europea & Unknown & 0 & 0 & 0 & 0 & 0 & 0 & 0 & \\
\hline 7 & Decile 1-3 & All Subjects & Accounting & Pasifika PecI & Male & 2 & 0 & 0 & 2 & 2 & 0 & 0 & \\
\hline 8 & Decile 1-3 & All Subjects & Accounting & Pasifika Pec & Female & 6 & 2 & 0 & 4 & 4 & 0 & 0 & \\
\hline 9 & Decile 1-3 & All Subjects & Accounting & \begin{tabular}{|l|l} 
Asian & I
\end{tabular} & Male & 5 & 0 & 0 & 5 & 5 & 0 & 0 & \\
\hline 10 & Decile 1-3 & All Subjects & Accounting & \begin{tabular}{|l|l} 
Asian \\
\end{tabular} & Female & 2 & 1 & 0 & 1 & 1 & 0 & 0 & \\
\hline 11 & Decile 1-3 & All Subjects & Accounting & Other/Unsp I & Male & 0 & 0 & 0 & 0 & 0 & 0 & 0 & \\
\hline 12 & Decile 1-3 & All Subjects & Accounting & Other/Unsp & Female & 0 & 0 & 0 & 0 & 0 & 0 & 0 & \\
\hline 13 & Decile 1-3 & All Subjects & Agricultural & NZ Maori & Male & 0 & 0 & 0 & 0 & 0 & 0 & 0 & \\
\hline 14 & Decile 1-3 & All Subjects & Agricultural & NZ Maori & Female & 0 & 0 & 0 & 0 & 0 & 0 & 0 & \\
\hline 15 & Decile 1-3 & All Subjects & Agricultural & NZ Europea I & Male & 0 & 0 & 0 & 0 & 0 & 0 & 0 & \\
\hline 16 & Decile 1-3 & All Subjects & Agricultural & NZ Europea & Female & 0 & 0 & 0 & 0 & 0 & 0 & 0 & \\
\hline 17 & Decile 1-3 & All Subjects & Agricultural & NZ Europea & Unknown & 0 & 0 & 0 & 0 & 0 & 0 & 0 & \\
\hline 18 & Decile 1-3 & All Subjects & Agricultural & Pasifika PecI & Male & 0 & 0 & 0 & 0 & 0 & 0 & 0 & \\
\hline 19 & Decile 1-3 & All Subjects & Agricultural & Pasifika Pec & Female & 0 & 0 & 0 & 0 & 0 & 0 & 0 & \\
\hline 20 & Decile 1-3 & All Subjects & Agricultural & Asian & Male & 0 & 0 & 0 & 0 & 0 & 0 & 0 & \\
\hline 21 & Decile 1-3 & All Subjects & Agricultural & Asian & Female & 0 & 0 & 0 & 0 & 0 & 0 & 0 & \\
\hline 22 & Decile 1-3 & All Subjects & Agricultural & Other/Unsp & Male & 0 & 0 & 0 & 0 & 0 & 0 & 0 & \\
\hline 23 & Decile 1-3 & All Subjects & Agricultural & Other/Unsp & Female & 0 & 0 & 0 & 0 & 0 & 0 & 0 & \\
\hline 24 & Decile 1-3 & All Subjects & Art History & NZ Maori & Male & 0 & 0 & 0 & 0 & 0 & 0 & 0 & \\
\hline 25 & Decile 1-3 & All Subjects & Art History & NZ Maori & Female & 2 & 0 & 0 & 2 & 2 & 0 & 0 & \\
\hline
\end{tabular}

${ }^{6}$ This Table is included in TableToLongForm as part of data(TCData), and can be accessed with TCData[["NZQAScholarships"]] 


\section{Diagnostics}

The primary limitation of TableToLongForm is that the function will be a black box to most users. After running the function on a Table, the user will either be given back a "data.frame" with no easy way of verifying if the result is correct, or be confronted with an error with little idea of what went wrong. Based on ad hoc tests conducted so far, TableToLongForm will either succeed, or fail catastrophically in a manner that is easily recognised as utter failure. However methods for verifying correct operation (or to understand failures) would be desirable.

The simplest method currently available is to examine the additional output returned when TableToLongForm is called with the optional argument fulloutput = TRUE. This will return the 'final product' of TableToLongForm's algorithms in the form of 'IdentResult', 'rowplist' and 'colplist'.

IdentResult was covered above in the User manual section and contains information on where the data and labels are found.

rowsplist and colsplist stand for Row/Column Parentage List which are nested "list" objects that represents all the hierarchical relationships in the Table, as identified by TableToLongForm. For easier reading they are assigned the "plist" class which has a custom 'print' method. An example of a 'colplist' is shown in Figure 5.

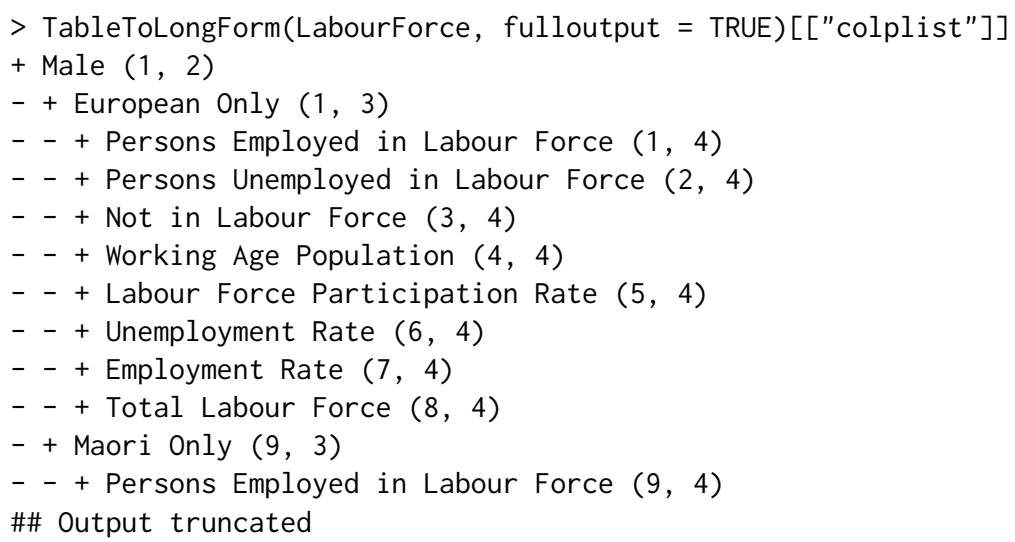

Figure 5: A truncated example of the 'colplist' for the Labour Force Status data used in Figure 1. It represents the hierarchical relationships of the column labels, as identified by TableToLongForm. We can see that it has correctly identified 'Male' as a top-level parent with the ethnic categories, such as 'European Only', nested inside, which are in turn a parent to the lowest-level categories, such as 'Employment Rate'.

Unfortunately this output has two key limitations. First, it is not obvious from this output what went wrong (or if nothing went wrong), requiring some detective work to piece together the evidence. Second, if anything did go wrong, the user still does not know why.

The option with the potential to provide the most information is calling TableToLongForm with the optional argument diagnostics = TRUE, which will write diagnostic output to a file, printing key variables at each major stage of the conversion process. This output can thus be used to track TableToLongForm's progress as it works to convert the Table, enabling fairly accurate assessment of where exactly it went wrong. Some example diagnostic output is shown in Figure 6. Unfortunately, understanding this output requires familiarity with the workings of the code and is unlikely to be of much use to anyone other than the author.

\section{Discussion}

This article has introduced TableToLongForm, an R package that can automatically convert hierarchical Tables that would normally rely on the discerning powers of a human brain, to a simple LongForm dataframe that any decent software package can easily manipulate and use. While it can handle a variety of Tables automatically, and an even greater variety with some aid from the human user, it is not without limitations. Ultimately, TableToLongForm still uses algorithms to detect a known set of recognised patterns and any Table that deviates from these patterns will break TableToLongForm.

There is work to be done in refining and generalising existing algorithms and creating new algorithms so that TableToLongForm can successfully handle more cases, while also reducing the 

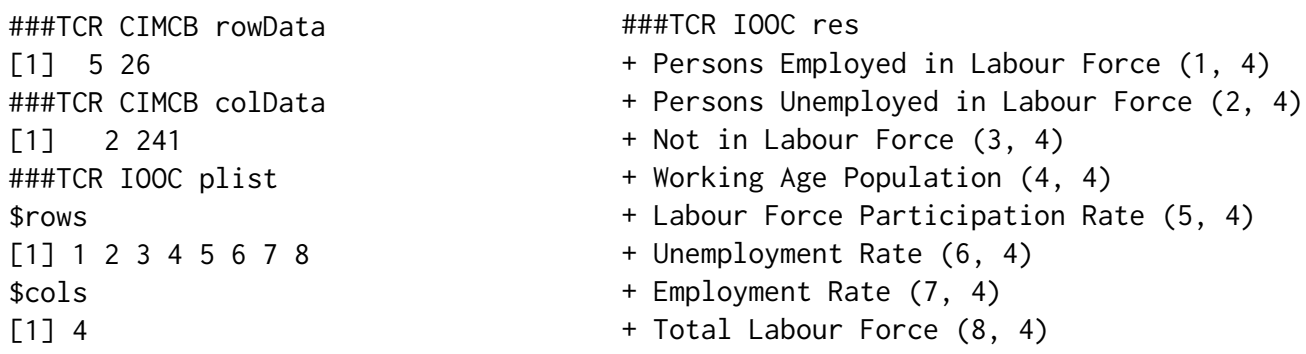

Figure 6: A few examples of the diagnostic output generated by TableToLongForm when called with 'diagnostics = TRUE' on the Labour Force Status data used in Figure 1. The diagnostic output is printing key variables at each major stage of the conversion process. '\#\#\#TCR' indicates an identifier line, the following word indicates the part of the function generating this output (e.g. 'CIMCB', which is short for Call Identification algorithm Most-Common-Boundary), the last word indicates the name of the variable being printed (e.g. 'rowData'). The diagnostic output can be used to see what TableToLongForm is getting right (or wrong)... assuming the user is familiar with the code.

possibility of a false positive. These range from adding more robust checks to the algorithms to verify correct detection, such as sum-checks or pattern recognition, to more fundamental changes, such as altering the "NA" classification to distinguish between empty space and missing values. A recent addition to the package (introduced in version 1.3.0) is to enable new, custom algorithms to be used in place of the default ones included with the package. More information on this can be found in the official webpage for the package.

There is also work to be done in diagnostics output, not only in the formal diagnostic output, but also in the form of error and warning messages. Consider for instance the following error message if we call TableToLongForm on the Table in Figure 3 without specifying the correct IdentResult. From these messages it is not at all obvious that the problem is an incorrect IdentResult, which is a problem that is relatively easy to address if only it can correctly be identified by the user.

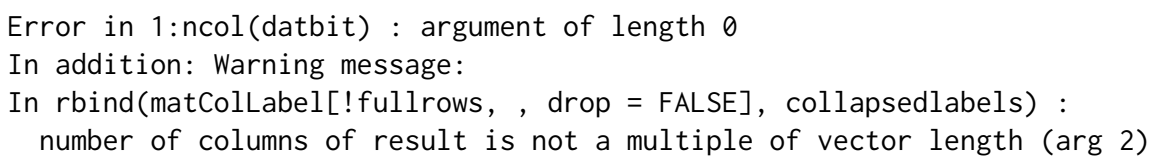

In terms of formal diagnostic output, various ideas are being tried such as graphical representations of the information provided by fulloutput = TRUE by drawing the original Table with the regions being highlighted in some way. Such a method would, for example, make it easier to see a problem with IdentResult, as it should become apparent on the drawn Table that the incorrect regions are being highlighted.

This article has been written for TableToLongForm version 1.3.1. The code for reproducing the figures in this article, as well as more detailed documentation on the code itself, can be found at https: //www. stat. auckland.ac.nz/ joh024/Research/TableToLongForm/. The development version can be found on github at https://github.com/joh024/TableToLongForm.

\section{Bibliography}

Department for Education (UK). NEET statistics quarterly brief: April to June, 2013. URL https://www.gov.uk/government/publications/neet-statistics-quarterly-brief-april-tojune-2013. [p19]

NZQA. Secondary School Statistics, 2012. URL http://www. nzqa.govt.nz/. [p23]

OpenRefine. OpenRefine, 2013. URL http://openrefine.org/. [p16]

Statistics New Zealand. Infoshare, 2013. URL http://www. stats.govt.nz/infoshare/. [p17]

I. Visne, A. Yildiz, E. Dilaveroglu, K. Vierlinger, C. Nöhammer, F. Leisch, and A. Kriegner. speedR: An R package for interactive data import, filtering and ready-to-use code generation. Journal of Statistical Software, 51(2):1-12, 2012. URL http://www. jstatsoft.org/v51/i02/. [p16]

H. Wickham. Reshaping data with the reshape package. Journal of Statistical Software, 21(12):1-20, 2007. URL http://www. jstatsoft.org/v21/i12/. [p16] 
H. Wickham. The split-apply-combine strategy for data analysis. Journal of Statistical Software, 40(1): 1-29, 2011. URL http://www. jstatsoft.org/v40/i01/. [p16]

H. Wickham. Tidy data. Journal of Statistical Software, 59(10), 9 2014. ISSN 1548-7660. URL http: //www. jstatsoft.org/v59/i10. [p16]

Jimmy Oh

Department of Statistics

The University of Auckland

New Zealand

joh024@aucklanduni.ac.nz 